\title{
artículos
}

\section{Los dibujos sobre lienzo de Manuel Mignorance Acién del decenio de 1980}

\author{
Enrique Castaños Alés y Antonia María Castro Villena \\ Universidad de Málaga
}

RESUMEN

La pasión por el dibujo de Manuel Mingorance y su deseo por ver consagrada la técnica dibujística como un arte independiente con prestigio similar al del temple o el óleo, determinará que en la década de los ochenta del pasado siglo, su obra alcance sus máximas posibilidades técnicas y plásticas.

PALABRAS CLAVE: Manuel Mingorance/ Dibujo/ Pintura siglo XX/Década de los 80 .

The Manuel Mingorance Acién's drawings on canvas of the decade of 1980

ABSTRACT

The passion for Manuel Mingorance's drawing and his desire for seeing the technology consecrated "dibujistica" as an independent art with prestige similar to that of the tempera or the oil, will determine that in the decade of the eighties of last century, his work reaches his maximum technical and plastic possibilities.

KEY WORDS: Manuel Mingorance/ Drawing/ XX th Paint/ Eighties.

Los primeros carboncillos sobre lienzo en la obra de Manuel Mingorance son del decenio de 1970, aunque no será hasta el decenio siguiente que esta técnica no alcance todas sus posibilidades técnicas y plásticas, como se puede comprobar especialmente en los cuadros de mayor formato, algunos de dos metros y medio de longitud. El origen de esta técnica en la producción del pintor está sobre todo relacionado con su pasión por el dibujo, esto es, por el deseo de ver consagrado el dibujo como una técnica independiente y con el mismo grado de prestigio que el temple o el óleo. Los miles de pequeños dibujos hechos por Mingorance tenían para él la limitación del tamaño. Necesitaba ver los dibujos plasmados en superficies mucho mayores, equiparados con la propia pintura. Cuando se decidió a acometerlos, el primer problema que se le planteó fue el del soporte. Como él mismo explica en su Tratado de mis materiales en la pintura, el papel no era el soporte más apropiado a tal fin, no sólo por la dificultad del manejo de papeles de grandes dimensiones, sino por la necesidad que conlleva de ser protegido mediante un cristal, con los casi inevitables reflejos que distorsionan la visión del espectador. De ahí que finalmente se decidiese por el lienzo, eligiendo para su primer ensayo una lona de algodón, por la unifor-

* CASTAÑos ALÉS, Enrique y CASTRO VILLENA, Antonia María:, "Los dibujos sobre lienzo de Manuel Mignorance Acién del decenio de 1980", en Boletín de Arte, n 30-31, Departamento de Historia del Arte, Universidad de Málaga, 2009-2010, págs. 479-486. Fecha de recepción: Diciembre de 2008. 

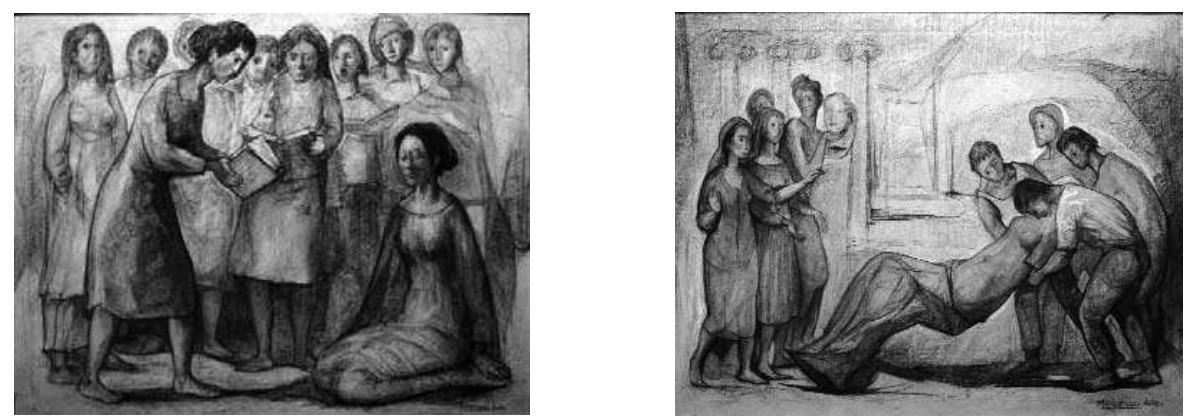

1. "Neófita", 1980.

2. "Renacimiento", 1980.

midad de su trama, soporte óptimo además, convenientemente preparado, para el empleo del carboncillo. La preparación de la lona, es decir, su imprimación, consistió en primer lugar en aplicar una mano de alkyl, encolado previo al aparejo. El alkyl es una cola sintética, industrial, que Mingorance prefiere a la tradicional cola de conejo porque ésta con la humedad tiende a hincharse y cuartea los cuadros. Una vez que se ha secado esta capa de encolado, el pintor prepara la imprimación propiamente dicha, que no es más que una mezcla de cemento blanco y escayola (lo que él llama «bolo blanco»), a la que se añade un poco de negro de marfil con el fin de convertir en gris la mezcla resultante («bolo gris»). La razón de obtener este color grisáceo no es otra que impedir la crudeza del blanco puro como fondo de los dibujos de mayor tamaño. La mencionada mezcla se une finalmente al alkyl, en la proporción de 1 parte de la mezcla en polvo de bolo gris por 1 1/2 de alkyl. Con esta proporción el aparejo es menos denso, impide la formación de surcos y no se anula la trama regular del tejido de lona, trama que ofrece condiciones idóneas para mantener el carboncillo cuando se dibuja con este material sobre ella.

Después de realizado el dibujo, Mingorance procede al fijado del carboncillo y al barnizado posterior. Ninguno de los fijativos ofrecidos por el mercado le han convencido nunca, razón por la que ha terminado elaborando uno de su propia invención, compuesto de una parte de alkyl, tres partes de agua y otra parte de alcohol puro. En los pequeños formatos emplea el pulverizador y en los cuadros de mayor tamaño la pistola con compresor. En cuanto al barniz, sustituye al cristal como protector, es lavable y no produce reflejos, es decir, termina formando parte del propio dibujo. Para aplicarlo, previamente, sobre la capa de fijativo, da un aislante, que no es más que un alkyl rebajado y que cuando se seca desaparece por completo. Sobre ese aislante seco se da finalmente el barnizado, un barnizado industrial, esto es, un 

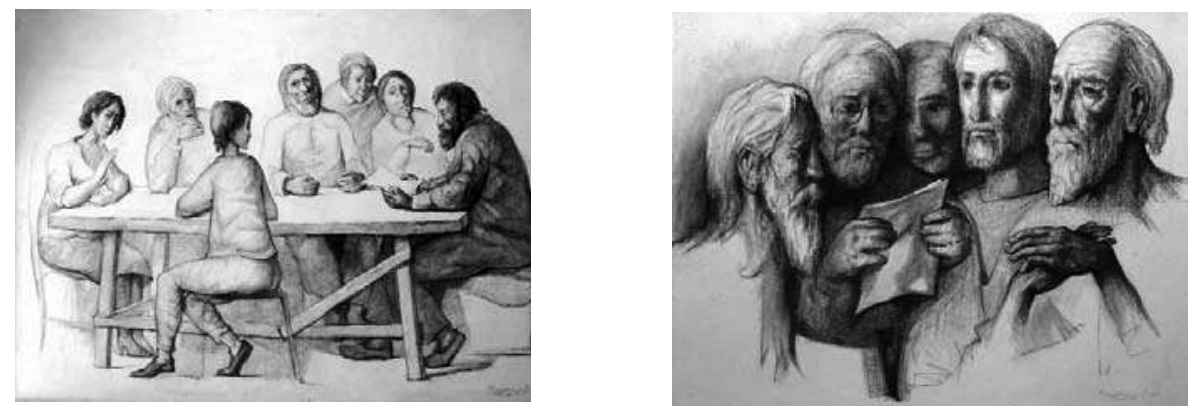

3. "La Secta", 1981.

4. "Aquí es donde dice que todos los hombres somos hermanos", 1985.

barnizado acrílico al agua, aplicado a pistola. Los años transcurridos han podido corroborar los excelentes resultados de todas estas técnicas, esenciales para analizar y estudiar uno de los capítulos más destacados de la producción de Mingorance, los dibujos sobre lienzo.

De los nueve dibujos que hemos seleccionado para estas anotaciones, los dos más antiguos están hechos con sanguina, un lápiz usado con frecuencia para este tipo de obras. Se trata de Neófita y de Renacimiento, ambos de 1980. En el primero de ellos, la composición se atiene a reglas clásicas: el grupo de figuras, inscrito en un rectángulo, tiene las cabezas situadas a la misma altura (isocefalia), como ya se hacía en los mosaicos bizantinos de Rávena en el siglo VI. El predominio de las verticales, con las figuras de pie, se ve contrarrestado y equilibrado por la muchacha neófita, sentada en posición arrodillada a la derecha, una de cuyas piernas marca la horizontal más pronunciada del cuadro. El elemento dinámico lo ofrece la figura inclinada de la izquierda, cumpliendo así con un procedimiento habitual en las composiciones del Quattrocento y de la pintura del Alto Renacimiento, por ejemplo en El tránsito de la Virgen de Mantegna (ca. 1461) y en Los Desposorios de Rafael (1504). En la Iglesia cristiana primitiva, los neófitos eran los recién bautizados, que durante ocho días llevaban una túnica blanca y no podían ser investidos de dignidad eclesiástica alguna. A aquélla parece pertenecer la joven recatada, con los ojos cerrados y las manos sobre las plantas de los pies, que está siendo instruida en los significados de los libros simbólicos, como diría el joven Hegel, por un grupo de seguidoras de la nueva fe.

El otro dibujo con sanguina, Renacimiento, también ofrece una composición muy lograda, con ambos grupos de figuras vinculados entre sí por la escultura que 

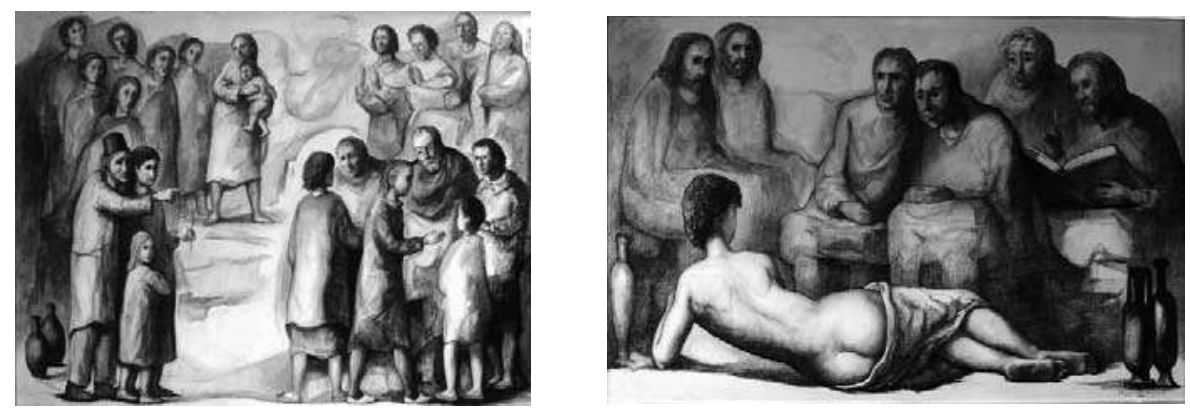

5. "Dicen que vendrá otra vez", 1982-83.

6. "Los filósofos y Friné", 1983.

ocupa el espacio central, que de otro modo quedaría demasiado vacío y desnudo. Este cuadro podría ser interpretado como una recreación de los hallazgos arqueológicos que se producían en Roma y en otras ciudades de Italia durante los siglos XV y XVI, tan importantes en el redescubrimiento de la Antigüedad clásica y en el surgimiento de la época artística y espiritual que conocemos con el nombre de Renacimiento. La figura que sostiene el grupo de muchachos, con los brazos cortados y el torso desnudo, nos evoca la Venus de Milo, pero también nos trae a la memoria esa efervescencia arqueológica de Roma a principios del siglo XVI, cuando Rafael Sanzio, a partir de abril de 1514, asume la responsabilidad de la superintendencia de los monumentos antiguos de Roma, que le convierte en la práctica, según ha explicado Sydney Joseph Freedberg, en el gran régisseur de las artes en Roma, con un estatus similar al de los ministros de la corte pontificia de León $X$. Indudablemente, las prolongadas estancias de Mingorance en la Ciudad Eterna han alimentado estas visiones.

Otro dibujo relacionado con Neófita es el que lleva por título La secta, de 1981 y de gran formato. Siete personas alrededor de una sobria mesa de madera, una de las cuales es una moza, escuchan atentamente la lectura de un documento que realiza uno de los miembros del grupo de iniciados, a todas luces pertenecientes a una secta religiosa. Pero repárese aquí en el modo en que se ha empleado el término secta, en el sentido latino de seguir una dirección o a un maestro. Es decir, no tanto en un sentido despectivo o peyorativo, como una desviación doctrinaria de la corriente principal, sino como un grupo embrionario, que con el tiempo puede terminar incluso convirtiéndose en Iglesia hegemónica. El ambiente espiritual que se describe es el que corresponde a los verdaderamente iniciados en una determinada corriente de pensamiento. Desde este punto de vista, también podría leerse como alusión al 
mundo filosófico más que al religioso. Pero en realidad, ambas parcelas parecen confundirse. El número siete, además, enfatiza el carácter propiamente religioso del grupo. Este número, que según explica el Diccionario de la Biblia de Herbert Haag y de A. van den Born debe su posición privilegiada en todo el mundo semítico a los siete días que dura cada una de las cuatro fases de la luna, tiene una amplia presencia en el Antiguo y en el Nuevo Testamento. En cualquier caso, se trata de personas pacíficas, entregadas sin ningún grado de fanatismo a su causa. Están predispuestas a escuchar, a debatir, a razonar las ideas.

De un contenido neotestamentario mucho más explícito son sendos dibujos titulados Dicen que vendrá otra vez (1982-83) y

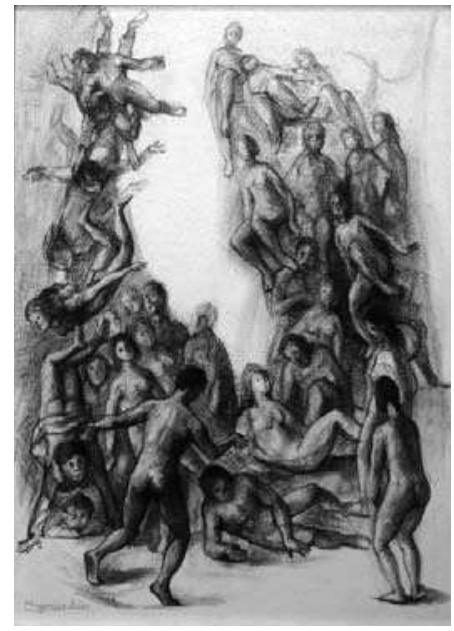

7. "Figuras emergidas", 1984. Aquí es donde dice que todos los hombres somos hermanos (1985). Este segundo cuadro, estrechamente relacionado con La secta, podría asimismo perfectamente representar a un reducido grupo de filósofos, de profetas o de sabios, y menos uno de ellos, que todavía es relativamente joven, los demás son hombres cargados de experiencia y que han vivido intensamente; de ahí su sosiego, su calma interior, la paz que inunda sus rostros. Están agrupados de manera compacta, en semicírculo, con semblantes graves, apropiados a la trascendencia del contenido del texto que lee uno de ellos mientras los otros escuchan. La primera formulación de una solidaridad o fraternidad universal entre todos los hombres, se remonta al pensamiento estoico antiguo, y de aquí influyó en el cristianismo. Esa fraternidad universal encuentra su fundamento en la idea de la totalidad orgánica del estoicismo, como puede advertirse en Crisipo de Cilicia. Dos cuerpos pueden interpenetrarse aunque sean de dimensiones diferentes. El «derecho natural» y la solidaridad entre todos los hombres en que piensa la Estoa, no excluye ni a los bárbaros, ni a los esclavos, ni a las mujeres, ni a los niños. La influencia de estas ideas en el mensaje evangélico es palpable, y en gran medida explica el grado de aceptación de la nueva religión cristiana entre las capas sociales más desfavorecidas del Imperio romano. La difusión de las ideas estoicas en Roma, en cuanto superación de los límites de la polis y la subsiguiente nueva concepción de la cosmópolis, también están en la raíz de la extensión de la ciudadanía a todos los habitantes del Imperio bajo Caracalla (212). Crisipo, que vive durante casi todo el siglo III a. C. y fue discípulo del fundador de la Escuela, Zenón de Citio, había escrito lo siguiente: «El mundo es un gran Estado con una constitución y una ley, a través de la cual 


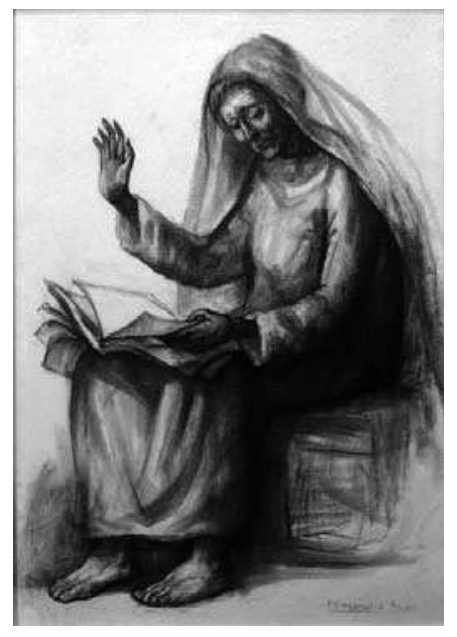

8. "Pitia Siracusana", 1984.

la razón natural ordena lo que hay que hacer y prohíbe lo que hay que omitir». Cuando en el Evangelio de San Juan leemos que Jesús se despide de sus discípulos después de la Última Cena diciendo: "Os doy un mandamiento nuevo: que os améis los unos a los otros. Que, como yo os he amado, así os améis también vosotros los unos a los otros», estamos ante la definitiva culminación de esa idea de la fraternidad universal, superada de modo paradójico e infinito por el propio sacrificio de quien lo dice. Esta sería, pues, otra segunda lectura del cuadro de Mingorance.

En lo que se refiere al gran lienzo denominado Dicen que vendrá otra vez, probablemente alude a la segunda venida de Cristo, anunciada por Jesús a sus discípulos al comienzo del capítulo 14 del Evangelio de San Juan. Pero del contenido de la escena no parece deducirse que el pintor se refiera al momento posterior a la Ascensión, pues los dos únicos evangelistas que tratan este asunto, San Marcos y San Lucas, nos dicen que la subida a los cielos fue sólo en presencia de los discípulos de Jesús. La misma precisión se hace al comienzo de los Hechos de los Apóstoles. Sin embargo, podría tratarse de una recreación de la dispersión de la multitud después del Sermón de la Montaña fusionada con la dispersión de los Apóstoles después de la Ascensión. Vemos juntos aquí adultos, ancianos, mujeres y niños, algunos con caras ensimismadas, otros comentando en corro, otros señalando y algunos rezando. El paisaje es muy sumario, casi inexistente, y a la izquierda vemos dos jarras apoyadas en el suelo que cierran la composición por ese lado. En cuanto a las figuras del fondo, por ejemplo la mujer que lleva a su hijo en brazos, están dibujadas y perfiladas con la misma nitidez que las del primer plano.

Una de las obras de gran formato más personales de la época que analizamos, es la que lleva por título Los filósofos y Friné (1983). Representa un célebre hecho legendario al que se refiere Marco Fabio Quintiliano en sus Instituciones oratorias (Libro II, capítulo XVI): «Créese también comúnmente que si se libró Friné no fue por la admirable defensa que de ella hizo Hipérides, sino porque ella, desabrochando la túnica, descubrió parte de su cuerpo, hermosísimo a la verdad». Acusada de asebeia, es decir, de menosprecio hacia la religión ateniense, la hetera Friné, que probablemente había sido la modelo de Praxíteles para la escultura de Afrodita venerada en Cnido, fue defendida por Hipérides ante la heliaía, el tribunal popular que, 
como instancia de apelación, era el más alto tribunal de justicia de los atenienses. El mito de Friné, que ha sido muy bien estudiado entre nosotros por el eximio jurista José Manuel Pérez-Prendes, gozó de cierta notoriedad entre los artistas academicistas y de estética decadente de los últimos decenios del XIX y principios del $\mathrm{XX}$, como lo revelan los casos del escultor holandés James Pradier y del pintor francés Jean-Léon Gérôme, aunque este último sitúa el episodio en un espacio cerrado, siendo bien sabido que los heliastas se reunían al aire libre. También Mingorance se ha tomado sus licencias, como llamar «filósofos» a quienes eran ciudadanos libres de Atenas. Pero la importancia de esta obra maestra radica en dos aspectos,

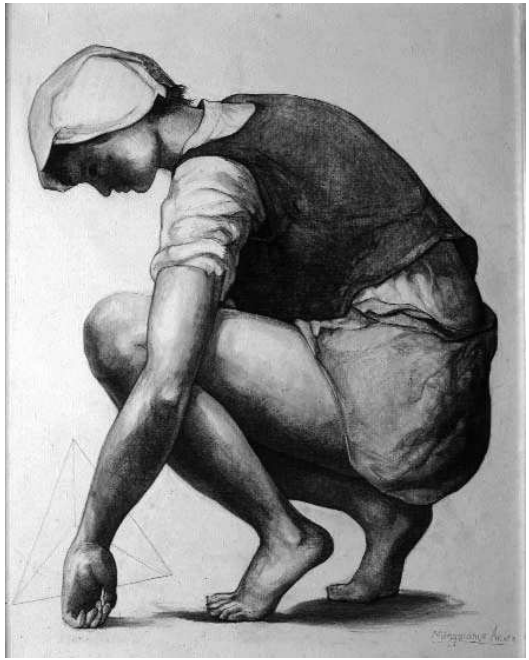

9. “Tétrada o Tetraedro", 1982. uno técnico y otro estético-espiritual. El

técnico se refiere al dibujo propiamente dicho. Maravillosamente modeladas las figuras, tanto las zonas iluminadas como las que permanecen en sombra definen con precisión los volúmenes, en un ejercicio dibujístico de resonancias clásicas e intemporales, como esa concepción platónica acerca de la belleza con la que tanto se identifica Mingorance. En cuanto al aspecto puramente espiritual, ha transmutado efectivamente a los jueces populares en «filósofos», graves y atentos, rebosantes de paz interior, que observan sin escandalizarse, porque en la Atenas del siglo IV antes de Cristo no podía ser de otro modo, la desnuda belleza del cuerpo de Mnésareté. En este cuadro se encierra el ideal de clemencia en la administración de la justicia en el que cree Mingorance.

Por último debemos referirnos a tres dibujos cuyos temas ofrecen diversas connotaciones estilísticas y simbólicas. En primer término, Figuras emergidas (1984), que no puede uno por menos de relacionarlas con las figuras de la zona izquierda inferior del Juicio Final de Miguel Ángel, en concreto la ascensión de los elegidos. En el célebre fresco de Buonarroti, los elegidos, de un estado casi de larvas en el que se encuentran una vez que se ha producido la resurrección de los muertos en el último día, ascienden trabajosamente hacia la gloria ayudados por otros bienaventurados, ofreciendo un catálogo de posiciones y de violentísimos escorzos verdaderamente inigualable. Algunos parecen quedar suspendidos en el espacio, en una región intermedia en la que permanecen como atrapados, pero finalmente consiguen la ayuda o el impulso ajeno suficiente para poder ascender a las alturas celestiales. En el cuadro de Mingorance unas figuras ascienden, las de la 
zona derecha, mientras otras caen y se precipitan, o, más bien, simulan planear y descender hasta llegar a tierra. En realidad se trata de un movimiento circular de ascensión y de caída, que quizás esté relacionado con el propio comportamiento humano y sus fluctuaciones en el terreno ético.

En segundo lugar, Pitia siracusana (1984), un nombre que no es el ortodoxo pero que podemos relacionar con la Sibila de Cumas, aquella a la que Apolo le había concedido el privilegio de vivir tantos años como granos de arena pudiese contener su mano, aunque a condición de no volver a Eritras, razón por la que se instaló en aquella ciudad de la Campania italiana. La leyenda más famosa cuenta que a medida que envejecía se iba volviendo más seca, hasta que finalmente la encerraron en una jaula que fue colgada en el templo de Apolo de Cumas. A la Sibila Cumana la representó Miguel Ángel en el techo de la Sixtina hojeando cansinamente el Libro de la sabiduría. También Mingorance la representa sentada y hojeando presumiblemente uno de los Libros sibilinos que fueron inspirados por ella. El arquetipo físico corresponde a los datos de la leyenda.

Para terminar, Tétrada o Tetraedro (1982), que puede ser interpretado como un comentario a uno de los jóvenes discípulos que atienden ensimismados las explicaciones de Euclides en el fresco de La Escuela de Atenas de Rafael. El autor de los Elementos dibuja con el compás una figura geométrica en una pizarra apoyada en el suelo, mientras que Mingorance ha preferido situar al fondo uno de los cinco poliedros regulares, el tetraedro. Estos poliedros, que tienen como caras polígonos regulares idénticos, que convergen en vértices idénticos, unidos por aristas de igual longitud y que son cada uno de ellos inscriptibles en una esfera, son también conocidos como cuerpos platónicos, por su importancia en la cosmografía de Platón. En el clásico estudio de Matila Costiesco Ghyca titulado Estética de las proporciones en la naturaleza y en las artes, se hace un concienzudo análisis de estos cuerpos, que han sido objeto de investigación y de estudio por diversos eruditos, sabios y artistas, como Piero della Francesca, quien les consagró su tratado Quinque Corporibus, dedicado al duque de Urbino en 1492, o Daniel Barbaro, Patriarca de Aquilea, en su Prattica de la Perspettiva (Venecia, 1569), o el astrónomo Johannes Kepler en su Mysterium Cosmographicum (1596). La figura de Mingorance, inscrita compositivamente en un rectángulo, se distingue por la corrección anatómica, la precisión de las articulaciones y el equilibrio de las líneas. 